\title{
Article
}

\section{Pressure-induced reconstructive phase transition in Cd3As2}

Gamza, Monika, Abrami, Paolo, Gammond, Lawrence V D, Ayres, Jake, Osmond, Israel, Muramtsu, Takaki, Armstrong, Robert, Perryman, Hugh, Daisenberger, Dominik, Das, Sitikantha and Friedemann, Sven

Available at https://clok.uclan.ac.uk/35107/

Gamza, Monika orcid iconORCID: 0000-0003-3360-4006, Abrami, Paolo, Gammond, Lawrence V D, Ayres, Jake, Osmond, Israel, Muramtsu, Takaki, Armstrong, Robert, Perryman, Hugh, Daisenberger, Dominik et al (2021) Pressure-induced reconstructive phase transition in Cd3As2. Physical Review Materials, 5 (2).

It is advisable to refer to the publisher's version if you intend to cite from the work. http://dx.doi.org/10.1103/PhysRevMaterials.5.024209

For more information about UCLan's research in this area go to http://www.uclan.ac.uk/researchgroups/ and search for <name of research Group>.

For information about Research generally at UCLan please go to

http://www.uclan.ac.uk/research/

All outputs in CLoK are protected by Intellectual Property Rights law, including Copyright law. Copyright, IPR and Moral Rights for the works on this site are retained by the individual authors and/or other copyright owners. Terms and conditions for use of this material are defined in the policies page.

\section{CLoK}

Central Lancashire online Knowledge www.clok.uclan.ac.uk

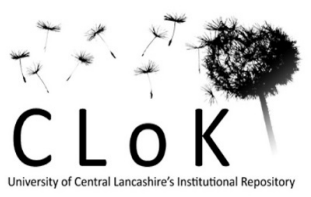




\title{
Pressure-induced reconstructive phase transition in $\mathrm{Cd}_{3} \mathrm{As}_{2}$
}

\author{
Monika Gamża $\odot,{ }^{1}$ Paolo Abrami, ${ }^{2}$ Lawrence V. D. Gammond $\odot,{ }^{2}$ Jake Ayres,${ }^{2}$ Israel Osmond, ${ }^{2}$ Takaki Muramatsu, ${ }^{2}$ \\ Robert Armstrong $\odot,{ }^{2}$ Hugh Perryman, ${ }^{2}$ Dominik Daisenberger, ${ }^{3}$ Sitikantha Das, ${ }^{2,4}$ and Sven Friedemann $\odot^{2, *}$ \\ ${ }^{1}$ Jeremiah Horrocks Institute for Mathematics, Physics and Astrophysics, University of Central Lancashire, \\ Preston PR1 2HE, United Kingdom \\ ${ }^{2} \mathrm{HH}$ Wills Laboratory, University of Bristol, Bristol, BS8 1TL, United Kingdom \\ ${ }^{3}$ Diamond Light Source, Harwell Science and Innovation Campus, Didcot, OX11 ODE, United Kingdom \\ ${ }^{4}$ Department of Physics, Indian Institute of Technology, Kharagpur, 721 302, India
}

(Received 5 October 2020; revised 22 January 2021; accepted 9 February 2021; published 26 February 2021)

\begin{abstract}
Cadmium arsenide $\left(\mathrm{Cd}_{3} \mathrm{As}_{2}\right)$ hosts massless Dirac electrons in its ambient-condition tetragonal phase. We report x-ray diffraction and electrical resistivity measurements of $\mathrm{Cd}_{3} \mathrm{As}_{2}$ upon cycling pressure beyond the critical pressure of the tetragonal phase and back to ambient conditions. We find that, at room temperature, the transition between the low- and high-pressure phases results in large microstrain and reduced crystallite size, both on rising and falling pressure. This leads to nonreversible electronic properties, including self-doping associated with defects and a reduction of the electron mobility by an order of magnitude due to increased scattering. This paper indicates that the structural transformation is sluggish and shows a sizable hysteresis of over $1 \mathrm{GPa}$. Therefore, we conclude that the transition is first-order reconstructive, with chemical bonds being broken and rearranged in the high-pressure phase. Using the diffraction measurements, we demonstrate that annealing at $\sim 200^{\circ} \mathrm{C}$ greatly improves the crystallinity of the high-pressure phase. We show that its Bragg peaks can be indexed as a primitive orthorhombic lattice with $a_{\mathrm{HP}} \approx 8.68 \AA, b_{\mathrm{HP}} \approx 17.15 \AA$, and $c_{\mathrm{HP}} \approx 18.58 \AA$. The diffraction study indicates that, during the structural transformation, a new phase with another primitive orthorhombic structure may also be stabilized by deviatoric stress, providing an additional venue for tuning the unconventional electronic states in $\mathrm{Cd}_{3} \mathrm{As}_{2}$.
\end{abstract}

DOI: 10.1103/PhysRevMaterials.5.024209

\section{INTRODUCTION}

Electronic states with topological properties have been observed in several materials in recent years [1]. Among these, $\mathrm{Cd}_{3} \mathrm{As}_{2}$ is particularly important, as it is stable in air and is easily synthesised via standard methods. The ambientcondition tetragonal phase of $\mathrm{Cd}_{3} \mathrm{As}_{2}$ hosts gapless electronic states with a linear dispersion relation caused by symmetryprotected band crossings along $\Gamma-Z$ in the Brillouin zone, as predicted by Wang et al. [2] and subsequently observed by angle-resolved photoemission spectroscopy and transport studies [3-6]. The topological protection leads to Fermi arcs on the surface of $\mathrm{Cd}_{3} \mathrm{As}_{2}$ crystals [7] and quantum Hall states along the edges $[8,9]$. Recently, topologically protected states have also been observed in thin films of $\mathrm{Cd}_{3} \mathrm{As}_{2}$ [10-12]. Thus, $\mathrm{Cd}_{3} \mathrm{As}_{2}$ is currently being explored for technological applications based on the topological properties, e.g., for optical switches, electronic devices operating at frequencies in the terahertz regime, and high-sensitivity photodetectors [13-15]. In addition, $\mathrm{Cd}_{3} \mathrm{As}_{2}$ is a prime candidate for applications based on the large magnetoresistance, room-temperature chiral charge pumping, thermoelectric properties, and possible topological superconductivity [16-20]. Assessing the stability of the ambient-condition phase of $\mathrm{Cd}_{3} \mathrm{As}_{2}$ and understanding

\footnotetext{
*sven.friedemann@bristol.ac.uk
}

conditions from which this phase can be recovered with ultrahigh carrier mobilities after applying strain is of paramount importance for the development of devices based on this topological semimetal.

The ambient-condition phase of $\mathrm{Cd}_{3} \mathrm{As}_{2}$ (phase I) hosting the topologically protected electronic excitations has a distorted superstructure of the $\mathrm{CaF}_{2}$ type [21]. Since $\mathrm{Cd}_{3} \mathrm{As}_{2}$ is $\mathrm{Cd}$ deficient compared with the ideal antifluorite stoichiometry, missing two out of eight $\mathrm{Cd}$ atoms needed to form a simple cube around the As, the superstructure can be envisioned as consisting of distorted $\mathrm{Cd}_{6} \square_{2}$ cubes (where $\square$ denotes an empty vertex) that are ordered in a spiraling corkscrew fashion along an axis parallel to the $c$ direction of the tetragonal crystal lattice. The latest single-crystal x-ray diffraction (XRD) study revealed that each corkscrew is surrounded by corkscrews of the opposite chirality, resulting in the centrosymmetric crystal structure with the space group $I 4_{1} /$ acd [21]. As a consequence of the centrosymmetric symmetry, no spin splitting is allowed at the symmetry-protected band crossings, and a bulk Dirac point at the Fermi energy along $\Gamma-Z$ results, making the ambient-condition phase of $\mathrm{Cd}_{3} \mathrm{As}_{2}$ a three-dimensional analog of graphene [22]. Breaking various symmetries using tuning parameters such as temperature or pressure, $P$, may drive this system into other nontrivial quantum states $[3,23,24]$.

With increasing temperature, $\mathrm{Cd}_{3} \mathrm{As}_{2}$ undergoes three consecutive structural phase transitions. It transforms to a 
$\mathrm{Zn}_{3} \mathrm{As}_{2}$-type structure $\left(P 4_{2} / n b c\right.$; phase II) at $\sim 220^{\circ} \mathrm{C}$ and to another primitive tetragonal phase of the $\mathrm{Zn}_{3} \mathrm{P}_{2}$-type $\left(P 4_{2} / n m c\right.$; phase III) at $\sim 470{ }^{\circ} \mathrm{C}$ before entering the $\mathrm{CaF}_{2}$ type face-centred cubic (fcc) structure $(F m \overline{3} m$; phase IV) at $\sim 600^{\circ} \mathrm{C}$, which remains stable to the melting temperature [25-27]. Crystal structures of the high-temperature phases can be viewed as antifluorite superstructures containing 128, 32, and 8 distorted $\mathrm{Cd}_{6} \square_{2}$ cubes per conventional unit cell, respectively, and the transitions between them are related to changes in distribution of $\mathrm{Cd}$ atoms among the tetrahedral voids [21]. In the high-temperature fcc phase, the $\mathrm{Cd}$ ions are disordered [26], whereas in all the lower-temperature variants, the $\mathrm{Cd}$ atoms form ordered patterns and shift from the ideal antifluorite positions toward the empty vertices of $\mathrm{Cd}_{6} \square_{2}$ cubes. The transition between the $\mathrm{Zn}_{3} \mathrm{As}_{2}-$ and $\mathrm{Zn}_{3} \mathrm{P}_{2}$-type phases at $470{ }^{\circ} \mathrm{C}$ has all the signatures of a typical first-order structural change; it proceeds quickly and with a notable thermal hysteresis. In contrast, the transition at $220^{\circ} \mathrm{C}$ from the $\mathrm{Zn}_{3} \mathrm{As}_{2}$-type structure to the ambient-temperature phase I progresses slowly and shows no distinct hysteresis [26]. Evidence for an intermediate phase with disordered crystal structure was found at the isomorphic transition in $\mathrm{Zn}_{3} \mathrm{As}_{2}$ [26].

At high pressures $(P)$, additional phases of $\mathrm{Cd}_{3} \mathrm{As}_{2}$ have been identified [25,27]. At room temperature, a high-pressure semiconducting phase $\mathrm{V}$ was reported with a trigonal, orthorhombic, or monoclinic symmetry [25,28-30] above $P_{c} \approx$ $2.3 \mathrm{GPa}$; this phase was mapped out to be stable up to $550^{\circ} \mathrm{C}$. Above $550{ }^{\circ} \mathrm{C}$, three more high-pressure phases were detected but have not yet been characterized. Raman and optical reflectivity measurements indicated the presence of a consecutive phase at pressures above 9.5 and $8 \mathrm{GPa}$, respectively $[31,32]$ where superconductivity was observed [30].

Although the presence of the $\mathrm{Cd}_{3} \mathrm{As}_{2}$ phase $\mathrm{V}$ at high pressures has been established, the correlation between the structural and electronic characteristics is still under debate. In particular, some studies show that the metal-to-semiconductor transition coincides with the structural phase transition from the ambient-condition phase I to the high-pressure phase $\mathrm{V}$ [29], whereas other reports indicate that the onset of semiconducting behavior occurs at much lower pressures $(\sim 1.1 \mathrm{GPa})$ compared with the structural phase transition [30]. Furthermore, recent magnetotransport measurements revealed a sudden shrinkage of the Fermi surface and an anomaly in the phase factor coincident with an anisotropic compression of the crystal lattice at $P \approx 1.3 \mathrm{GPa}$, interpreted as an indication for opening of a band gap [33]. Those inconsistent findings motivated us to investigate in detail the pressure-driven evolution of the crystal structure and transport properties of $\mathrm{Cd}_{3} \mathrm{As}_{2}$ between the ambient-condition phase I and the high-pressure phase $\mathrm{V}$ by means of high-resolution powder XRD, electrical resistivity, and Hall effect measurements.

\section{METHODS}

\section{A. Experimental details}

Needle-shaped single crystals were grown from self-flux in Cd excess, as described in Ref. [21]. High-purity $\mathrm{Cd}$ and As were loaded into an alumina crucible and sealed inside a quartz ampoule. The materials were heated to $825^{\circ} \mathrm{C}$ and slowly cooled to $425^{\circ} \mathrm{C}$ before being centrifuged to remove excess $\mathrm{Cd}$. The single-crystal nature of selected samples was confirmed with XRD. Stoichiometric composition of representative single crystals was confirmed with energy dispersive X-ray spectroscopy. Our samples show a residual resistivity ratio of seven, slightly larger than samples in previous high-pressure studies [29,30,33]. As-grown single crystals displaying clear facets were used for our electrical transport study. For powder XRD measurements, crystals were ground with a slight amount of glycerol to avoid dust.

Electrical resistivity and Hall effect measurements at room temperature were conducted in piston-cylinder cells with glycerol as a pressure transmitting medium. The resistance of a manganin wire was used as a continuous pressure gauge with in situ calibration on the resistive transitions of bismuth I-II and II-III [34]. The load on the piston-cylinder cell was regulated with a home-build hydraulic setup enabling slow pressure sweeps with virtually constant sweep rate $d P / d t$ (see inset of Fig. 2). The resistance and Hall effect were measured using a standard 4-point technique with a Stanford-Research SIM921 ac resistance bridge. The Hall effect was measured at fixed pressures in a $\pm 0.2 \mathrm{~T}$ electromagnet, giving rise to a transverse resistance $R_{t}$ linear in magnetic field $B$. The Hall coefficient $R_{H}$ was extracted from the slope of linear fits to $R_{t}(B)$. Sample dimensions were measured with an optical microscope; the uncertainty of the thickness measurement propagates to a systematic uncertainty of $\pm 15 \%$ in $R_{H}$.

High-pressure powder XRD measurements were performed in membrane-driven diamond anvil cells (DACs) at the $\mathrm{I} 15$ beamline at Diamond Light Source. X-ray radiation with wavelength $\lambda=0.4246(5) \AA$ was used throughout as calibrated on $\mathrm{a} \mathrm{LaB}_{6}$ standard. Pressure was measured using the ruby florescence method [35]. Diffraction peaks of residual cadmium flux mixed with $\mathrm{Cd}_{3} \mathrm{As}_{2}$ were used as a second pressure marker, thus allowing us to assess errors in the measured pressure values, as shown in Fig. 1. Three sample loadings were prepared and are labeled as sample 1, sample 2, and sample 3. For the first two loadings, glycerol was used as a pressure medium, whereas sample 3 was immersed in silicon oil. Temperatures up to $250{ }^{\circ} \mathrm{C}$ have been accessed with an external heater for samples 2 and 3. Diffraction patterns were acquired on a MAR345 image plate detector and integrated with the DAWN software [36] using the calibration parameters obtained from the $\mathrm{LaB}_{6}$ standard.

\section{B. Analysis of XRD patterns}

Refinements of the diffraction patterns were carried out with Jana2006 [38]. Profile refinements using the Le Bail method were used for evaluating the structural parameters and for performing strain and size analyses. Contributions to the diffraction peak broadening originating in grain size and microstrain were separated based on their different dependencies on the scattering angle $\theta$. To this end, reflections visible in diffraction patterns in the $2 \theta$ range from $6^{\circ}$ to $\sim 16^{\circ}$ were fitted using Lorentzian and pseudo-Voigt shape functions [39]. The variations of the Lorentzian and Gaussian half widths with 


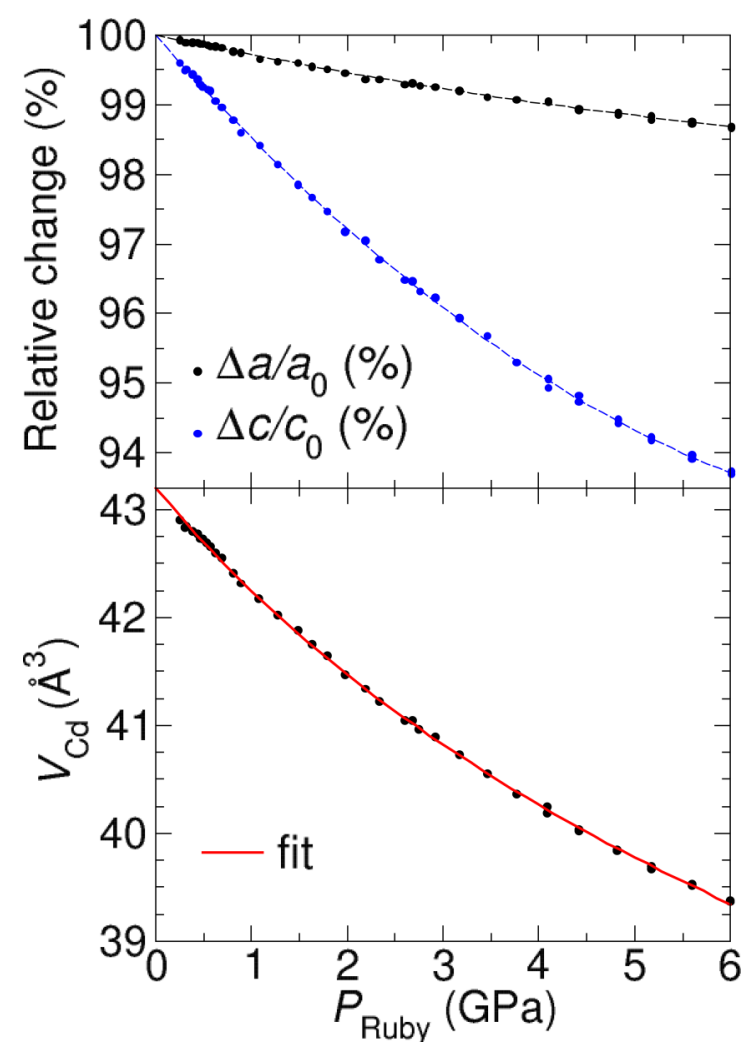

FIG. 1. Unit cell volume of $\mathrm{Cd}$ (bottom panel) and relative changes in its lattice parameters (top panel) obtained from Le Bail refinements of powder XRD patterns recorded on sample 1 at room temperature and plotted as a function of pressure measured using ruby fluorescence. Dashed lines are guides to the eye. Solid red line is the fit to the experimental data points using the Murnaghan equation of state with $K_{0}=43 \mathrm{GPa}, K_{0}^{\prime}=8$, and $V_{0}=43.2 \AA^{3}$, in agreement with Ref. [37]. Error bars in the pressure values evaluated based on the deviations of the fit from the data points do not exceed $0.05 \mathrm{GPa}$.

Bragg angle $\theta$ are described by the equations

$$
H_{L}=\frac{X}{\cos \theta}+Y \tan \theta+\Gamma_{L}(\theta)
$$

and

$$
H_{G}^{2}=8 \ln 2\left[\frac{P}{\cos ^{2} \theta}+U \tan ^{2} \theta+\Gamma_{G}^{2}(\theta)\right] .
$$

In each of Eqs. (1) and (2), the first term accounts for isotropic Scherrer particle broadening, the second term describes microstrain broadening related to lattice defects, while the last term stands for the Lorentzian $\left(\Gamma_{L}\right)$ and Gaussian $\left(\Gamma_{G}\right)$ parts of instrumental broadening. The latter was assessed from powder XRD pattern of virtually strainfree $\mathrm{LaB}_{6}$ powder with controlled grain size in the range of tens of micrometers. In the refinements, only one term describing grain size effect and one term describing microstrain broadening were used throughout.

According to the Scherrer formula $[40,41]$, the volume weighted crystallite size $D_{V}$ is given by

$$
D_{V}=\frac{K \lambda}{\beta \cos \theta},
$$

where $K$ denotes the Scherrer constant and is assumed to be 1 , $\lambda$ is the wavelength of the radiation used for the XRD study, while $\beta$ represents integral breadth of a reflection located at $2 \theta$ expected, assuming that the broadening originates solely in the grain size effect [42]. Therefore, the volume averaged size of crystallites can be calculated from refined $X$ or $P$ values in degrees using the formulas

$$
D_{V, L}=\frac{360 K \lambda}{\pi^{2} X} \quad \text { and } \quad D_{V, G}=\frac{180 K \lambda}{\sqrt{2 \pi^{3} P}},
$$

for Lorentzian and Gaussian-type broadening attributed to the grain size effect, respectively. The microstrain is given by

$$
\epsilon=\frac{\beta}{\tan \theta} .
$$

Therefore, the respective Lorentzian and Gaussian contributions are determined as

$$
\epsilon_{L}=\frac{3 \pi^{2} Y}{1440} \quad \text { and } \quad \epsilon_{G}=\frac{\sqrt{2 \pi^{3} U}}{720} .
$$

XRD data for the high-pressure phase of $\mathrm{Cd}_{3} \mathrm{As}_{2}$ was also analyzed by combining a Williamson-Hall method [43] with line profile analysis. To this end, a profile fitting procedure was adopted in which the measured XRD patterns were decomposed into individual peaks described using a pseudo-Voigt or Lorentzian shape function. According to the Williamson-Hall equation:

$$
\beta \cos \theta=\frac{K \lambda}{D_{V}}+4 \epsilon \sin \theta .
$$

Therefore, the microstrain $\epsilon$ and the volume-weighted crystallite size $D_{V}$ are calculated from the slope and the intercept of the plot of $\beta \cos \theta$ against $\sin \theta$ obtained after correcting integral breadths of the diffraction peaks for the instrumental broadening, assuming $K=1$.

Indexing of powder XRD patterns was performed using McMaille [44] and DICVOL06 [45] computer codes. Profile decomposition of the measured XRD patterns gave angular positions and intensities of individual peaks which were used as an input. Searching for candidate crystal lattices with volumes of up to $6000 \AA^{3}$ was carried out in all crystal systems by means of Monte Carlo algorithms [44] or an exhaustive trial-and-error method with variation of parameters by successive dichotomy and partitioning of the unit cell volume [45]. Space groups for the resulting sets of lattice parameters were identified using JANA2006 [38].

\section{EXPERIMENTAL RESULTS}

We start by presenting continuous resistivity as well as discrete Hall effect measurements in Sec. IIIA, showing the hysteresis and nonreversible behavior of the electronic properties upon pressure cycling of $\mathrm{Cd}_{3} \mathrm{As}_{2}$ into phase $\mathrm{V}$ and back to low pressure. Subsequently, we introduce the XRD results in Sec. IIIB, which demonstrate the reversion to the ambientpressure tetragonal phase I and show poor crystallinity after the pressure cycling. Together, these measurements provide evidence for a first-order reconstructive phase transition. We evaluate the compressibility of the low- and high-pressure phases of $\mathrm{Cd}_{3} \mathrm{As}_{2}$ at room temperature. We show that 
annealing of the high-pressure phase at $\sim 200{ }^{\circ} \mathrm{C}$ greatly improves its crystallinity and thus allows us to reveal more details about its crystal lattice. Finally, we indicate the formation of a new primitive orthorhombic phase prompted presumably by deviatoric stress upon releasing pressure through the structural phase transition in $\mathrm{Cd}_{3} \mathrm{As}_{2}$.

\section{A. Electrical transport}

As a crosscheck, we first measured the resistance of $\mathrm{Cd}_{3} \mathrm{As}_{2}$ on compression and decompression while staying within phase I at room temperature. Using a pristine single crystal, the pressure was first increased to $2 \mathrm{GPa}$ and subsequently released back down to $0.5 \mathrm{GPa}$. As shown by gray lines in Fig. 2(a), $\rho(P)$ is fully reversible within phase I and increases gradually with pressure.

Once cycling the pressure through the phase transition into the $\mathrm{Cd}_{3} \mathrm{As}_{2}$ phase $\mathrm{V}$ at room temperature, the resistance of $\mathrm{Cd}_{3} \mathrm{As}_{2}$ shows large hysteresis and nonreversible behavior. Subsequent to the cycling at low pressures, the pressure has been ramped to $3 \mathrm{GPa}$ and released back down to $0.5 \mathrm{GPa}$, as shown by the red data in Fig. 2(a). The transition from phase I to phase $\mathrm{V}$ is clearly seen as a pronounced steplike increase in the resistance with a sharp onset at $P_{c \uparrow}=2.3 \mathrm{GPa}$. Above $2.6 \mathrm{GPa}$, the resistance follows again a linear increase with further increasing pressure. The small linear increase below $P_{c \uparrow}$, the jump at $P_{c \uparrow}$, and the steeper linear increase above $P_{c \uparrow}$ are in very good agreement with earlier studies [29,30]. Upon releasing the pressure, the resistance follows this linear regime to well below $P_{c \uparrow}$, indicating a large hysteresis of the phase transition. Only at $P_{c \downarrow}=1 \mathrm{GPa}$, a large rise and sharp cusp is observed, indicating a phase transition. Most notably though, the resistance does not return to the low-pressure value. We note that the linear behavior observed below $P_{c \downarrow}$ after pressure cycling extrapolates to a value more than double the starting value, yet we show below in our analysis of the x-ray measurements that this second transition at $P_{c \downarrow}$ is associated with the reverse transition from phase $\mathrm{V}$ to phase I. All characteristics of the pressure-dependent resistance have been reproduced in three samples from the same batch.

Our Hall effect measurements at room temperature indicate a large increase in charge carrier concentration in the highpressure phase of $\mathrm{Cd}_{3} \mathrm{As}_{2}$. The results shown in Fig. 2(b) were obtained upon stepwise increasing the pressure to $3 \mathrm{GPa}$ and subsequently releasing it down to $0.5 \mathrm{GPa}$ at room temperature. While raising the pressure, the Hall coefficient remains roughly constant at $R_{H}=4.5 \times 10^{-6} \mathrm{~m}^{3} \mathrm{C}^{-1}$, in qualitative agreement with previous measurements at $2 \mathrm{~K}$ [33]. We note that the systematic difference by a factor of 3 and 10 with earlier studies by He et al. [6] and Zhang et al. [33], respectively, is probably due to uncertainties in the sample thickness and/or different levels of doping while the difference between our room temperature and the previous low-temperature measurements is small [6]. The qualitative agreement continues at high pressures where, above $P_{c \uparrow}$, the Hall coefficient is reduced to $R_{H}=1.6 \mathrm{~m}^{3} \mathrm{C}^{-1}$. In a simple single-band model, the drop of $R_{H}$ at $P_{c \uparrow}$ corresponds to a threefold increase of the charge carrier concentration $n$ in the high-pressure phase. This is similar to the sixfold increase of $n$ observed at $2 \mathrm{~K}$ by Zhang et al. [33]. Together with the increase in resistance above $P_{c \uparrow}$, this suggests an order of magnitude reduction of the
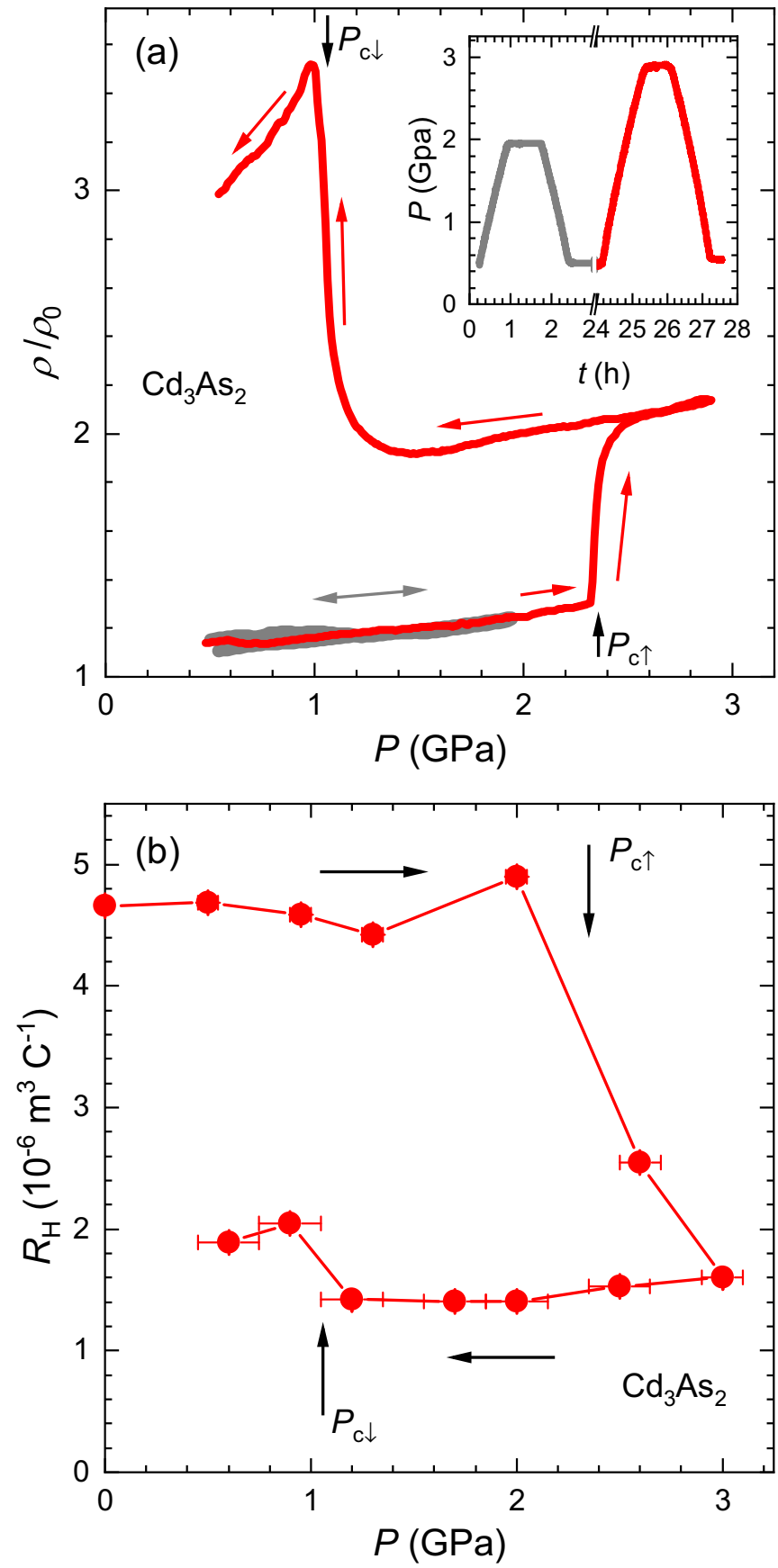

FIG. 2. (a) Nonreversible room-temperature resistivity $\rho / \rho_{0}$ where $\rho_{0}=\rho(P=0)$ and (b) Hall effect under pressure in $\mathrm{Cd}_{3} \mathrm{As}_{2}$. The initial pressure cycle from ambient pressure to $2 \mathrm{GPa}$ carried out on a pristine sample is shown in gray. It was followed by the second pressure cycle to $2.9 \mathrm{GPa}$ and back down to $0.5 \mathrm{GPa}$, shown in red. Horizontal arrows indicate the evolution of pressure, vertical arrows highlight the position of the critical pressure on increasing pressure $\left(P_{c \uparrow}\right)$ and decreasing pressure $\left(P_{c \downarrow}\right)$. Inset illustrates the pressure protocol for the two cycles.

carrier mobility in the high-pressure phase, as also observed by Zhang et al. [33].

The initial value of $R_{H}$ is not recovered after the pressure cycling. On decreasing pressure, the Hall coefficient remains roughly constant down to $P_{c \downarrow}$, with only a small increase 


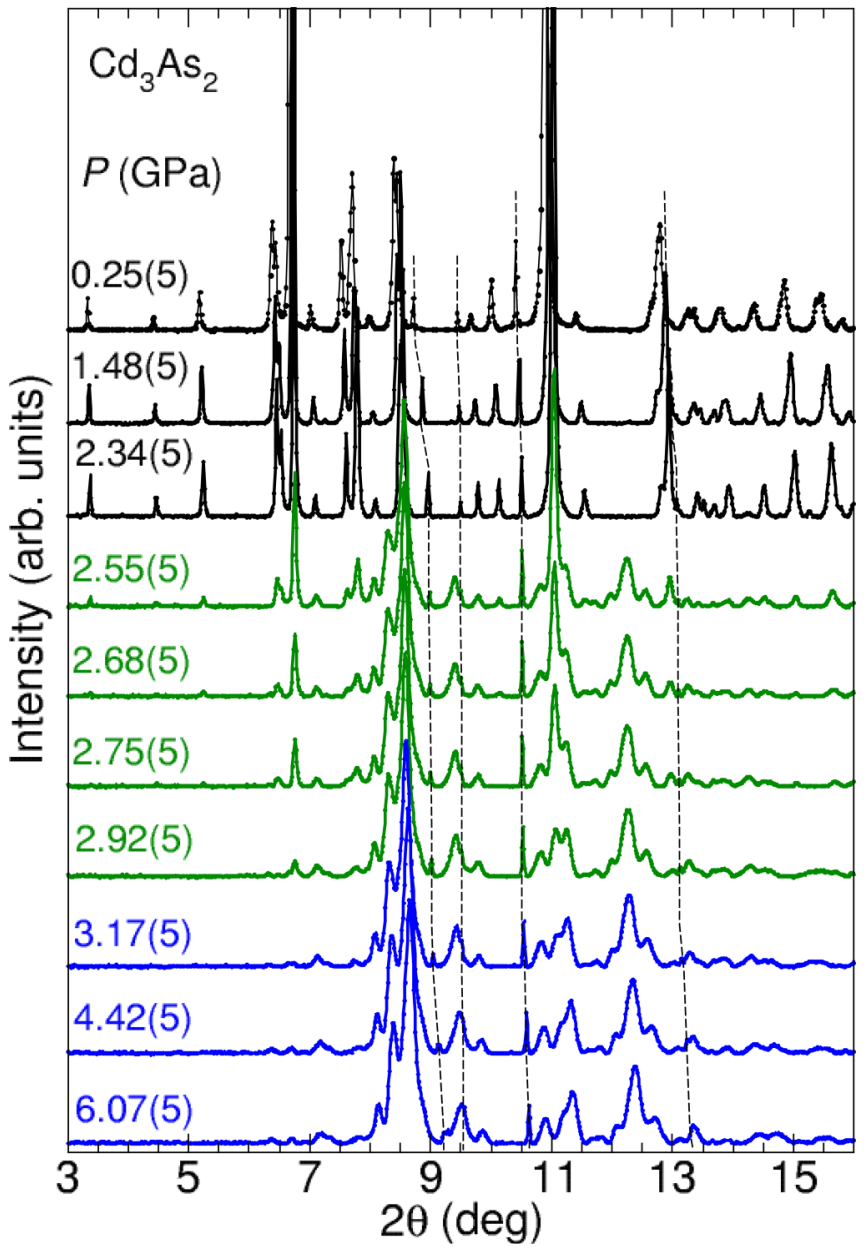

FIG. 3. Evolution of the XRD pattern recorded for $\mathrm{Cd}_{3} \mathrm{As}_{2}$ at room temperature in sequence proceeding from top to bottom, i.e., pressure raised from 0.25(5) GPa to 6.07(5) GPa. Diffraction patterns consistent with the ambient-condition phase I are shown in black, while blue color is used for the XRD data corresponding to the highpressure phase V. Diffraction patterns in green contain Bragg peaks originating in both the low- and high-pressure phases of $\mathrm{Cd}_{3} \mathrm{As}_{2}$, thus implying coexistence of phases I and $\mathrm{V}$ at pressures between $2.45(10)$ and $\sim 3 \mathrm{GPa}$. Dashed vertical lines indicate positions of peaks originating in $\mathrm{Cd}$.

below $P_{c \downarrow}$ to $R_{H}=2 \mathrm{~m}^{3} \mathrm{C}^{-1}$, a value close to early measurements of polycrystalline samples [46]. The irreversible behavior in $\rho(P)$ and $R_{H}(P)$ suggests either the transformation into a phase different than the original ambient-condition phase I or fundamentally modified electronic behavior like increased scattering and/or charge carrier doping. To explore the origin of the nonreversibility in the resistivity and Hall coefficient, we performed detailed powder XRD measurements.

\section{B. X-ray diffraction}

Our XRD study on a pristine sample of the ambientcondition phase of $\mathrm{Cd}_{3} \mathrm{As}_{2}$ after loading it into the pressure cell indicate that the powder was well crystalline and single phased, except for a small contribution of cadmium flux detected as characteristic peaks at $2 \theta \simeq 8.8^{\circ}, 9.5^{\circ}, 10.4^{\circ}$, and $16.2^{\circ}$ (see Fig. 3), and used as a second pressure marker
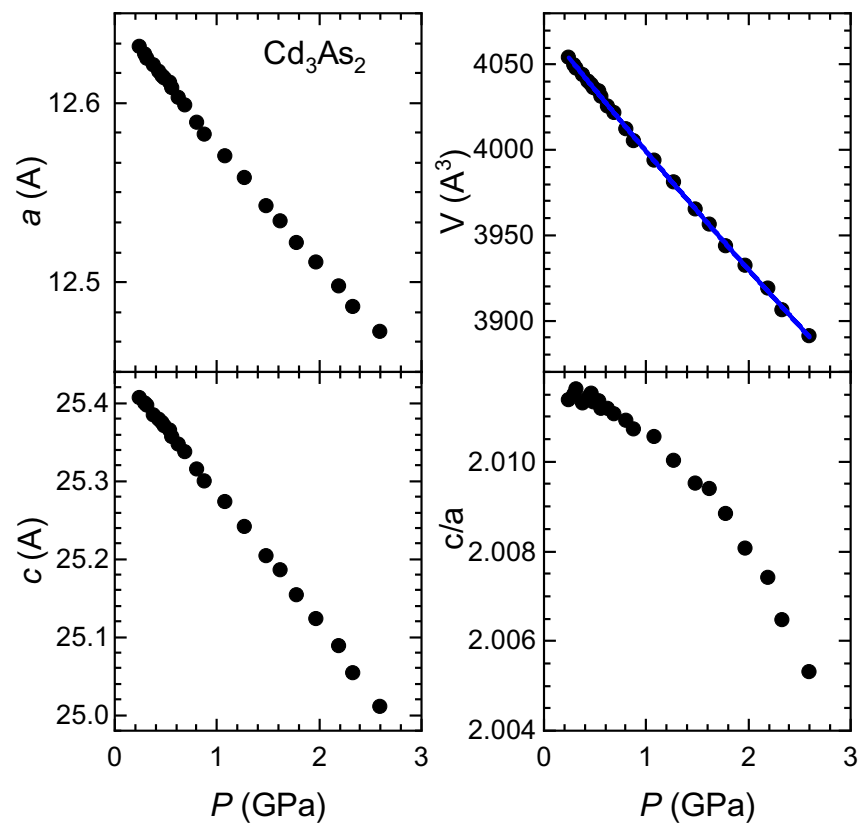

FIG. 4. Lattice parameters, their ratio, and the conventional unit cell volume of the ambient-condition phase of $\mathrm{Cd}_{3} \mathrm{As}_{2}$ at room temperature plotted as a function of pressure. Error bars are smaller than the size of the circles. Solid blue line is the fit to the experimental data points using the Murnaghan equation of state.

(see Fig. 1). To evaluate microstrain and sizes of $\mathrm{Cd}_{3} \mathrm{As}_{2}$ crystallites, Le Bail refinements of powder XRD patterns collected from several different sample spots were carried out. Excellent fits to the measured XRD data were achieved using pseudo-Voigt peak profiles with Gaussian contribution describing broadening due to the average grain size $D_{V}=$ $170(30) \mathrm{nm}$, and with Lorentzian distribution accounting for microstrain $\epsilon=0.24(2) \%$. The rather significant positive microstrain indicates the presence of local compressive strain fields. They were likely created when dissipating mechanical energy during grinding single crystals of $\mathrm{Cd}_{3} \mathrm{As}_{2}$ into a fine powder suitable for the powder XRD study. The refined lattice parameters of the $\mathrm{Cd}_{3} \mathrm{As}_{2}$ phase $\mathrm{I}[a=12.6303(2) \AA$, $c=25.409(1) \AA$, see Fig. 4] are very close to those reported in previous studies $[21,33,47]$.

Figure 3 shows the evolution of XRD patterns collected under pressure at room temperature. With increasing pressure, the diffraction peaks are gradually shifted to larger diffraction angles, indicating a pressure-induced shrinkage of the unit cell. For pressures up to $P_{c \uparrow}$, the reflection pattern is consistent with the ambient-condition tetragonal phase I of $\mathrm{Cd}_{3} \mathrm{As}_{2}$. The widths and shapes of all the peaks remain virtually the same compared with immediately after closing the pressure cell, indicating an elastic response of the crystal lattice to the applied stress field and giving no evidence for stress heterogeneity which would otherwise result in broadening of diffraction peaks. Lattice parameters derived from Le Bail refinements of the XRD patterns are shown in Fig. 4. The pressure-induced changes in the unit cell volume are well described by the Murnaghan equation of state fit, resulting in a bulk modulus $K_{0}=54(1) \mathrm{GPa}$, the pressure derivative of the bulk modulus $K_{0}^{\prime}=2.3(8)$, and the ambient pressure 


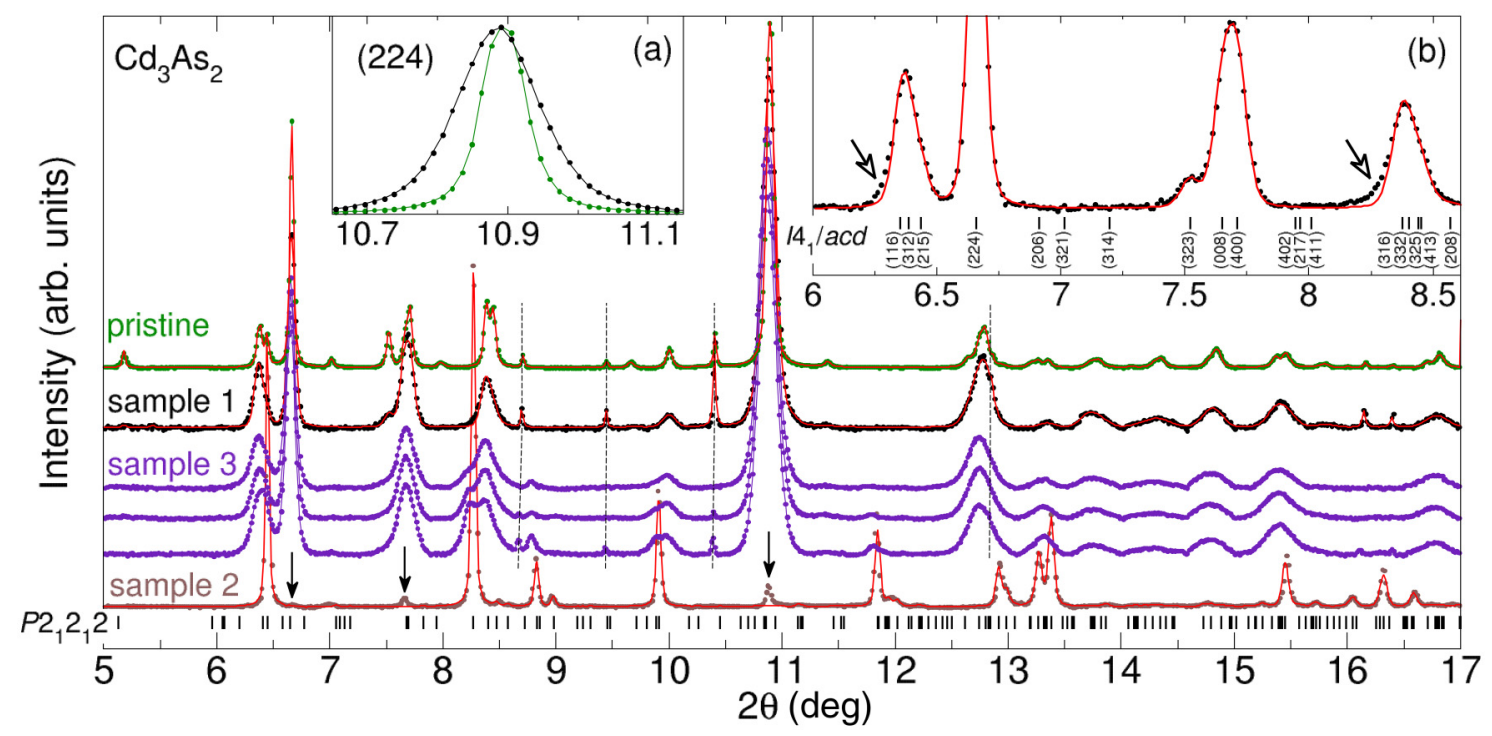

FIG. 5. Powder XRD patterns recorded at room temperature on $\mathrm{Cd}_{3} \mathrm{As}_{2}$ before [green dots, $P=0.25(5) \mathrm{GPa}$ ] and after the pressure cycling. During the pressure cycling, the high-pressure phase was either kept at room temperature [sample 1: black dots, $P=0.18(5) \mathrm{GPa}$ ] or was annealed at temperatures up to $240^{\circ} \mathrm{C}$ and cooled down to room temperature before pressure was released [sample 3: purple dots, $P=0$; sample 2: beige dots, $P=0.6(1) \mathrm{GPa}$. Solid red line on top of the XRD data for sample 2 represents Le Bail refinement performed assuming pseudo-Voigt profiles for diffraction peaks consistent with an orthorhombic lattice with the space group $P 22_{1} 2$ and the lattice parameters $a_{\mathrm{IP}}=8.026(2) \AA, b_{\mathrm{IP}}=4.1145(5) \AA$, and $c_{\mathrm{IP}}=18.988(1) \AA$. Angular positions of diffraction peaks expected for this orthorhombic lattice are indicated by short vertical black lines. Solid red lines on top of the XRD patterns for samples 1 and pristine correspond to Le Bail refinements performed assuming the ambient-condition phase I crystal lattice. Vertical arrows mark peaks attributed to a small amount of the ambient-condition phase in sample 2. Dashed vertical lines indicate positions of diffraction peaks originating in Cd. Inset (a) shows the (224) reflection of the tetragonal $I 4_{1} /$ acd phase $\mathrm{I}$ of $\mathrm{Cd}_{3} \mathrm{As}_{2}$ before and after the pressure cycling performed at room temperature, normalized to the maximum intensity. Inset (b) shows enlarged angular range in which a pronounced asymmetric broadening is seen for some Bragg peaks of the ambient-condition phase of $\mathrm{Cd}_{3} \mathrm{As}_{2}$ recovered after the room temperature pressure cycling. Short black lines in (b) indicate positions of reflections compatible with the crystal structure of the ambient-condition phase.

volume $V_{0}=4072(1) \AA^{3}$. The bulk modulus of the ambientcondition phase of $\mathrm{Cd}_{3} \mathrm{As}_{2}$ is smaller than that of copper and comparable with elemental $\mathrm{Cd}$.

At pressures above $\sim 2.5 \mathrm{GPa}$, a clear alteration of the XRD pattern is observed (see Fig. 3). This includes the loss of many peaks, e.g., for $2 \theta \leqslant 6^{\circ}$, and the emergence of new peaks incompatible with the space group $I 4_{1} /$ acd of the phase I, e.g., at $2 \theta \simeq 12.5^{\circ}$, implying a change in the symmetry of the crystal lattice. The transition progresses slowly, with intensities of diffraction peaks originating in the low- and high-pressure phases changing gradually in the pressure range from $\sim 2.5$ to $\sim 2.9 \mathrm{GPa}$ and thus signifying a distinct phase coexistence regime.

The diffraction pattern of the high-pressure phase shown in Fig. 3 is similar to those reported in [29,30,33], where it was interpreted as a monoclinic structure. All the diffraction peaks of the high-pressure phase appear very broad. At the same time, the small width of reflections originating from residual $\mathrm{Cd}$ flux highlights the high-quality pressure conditions with negligible pressure gradients. Thus, we conclude that the broadening of the $\mathrm{Cd}_{3} \mathrm{As}_{2}$ reflections is intrinsic to the sample. Since at low $2 \theta$ the effect of microstrain on widths of peaks is insignificant, such a huge broadening even at lowest diffraction angles indicates that, during the pressure-induced structural phase transition, the hundreds of nanometer-sized grains observed in the pristine phase I form much smaller domains in the high-pressure $\mathrm{Cd}_{3} \mathrm{As}_{2}$ phase $\mathrm{V}$, presumably due to local strains arising from enhanced crystalline defects. Estimates for the volume-weighted domain size performed using Eq. (3) based on integral breaths of the first four reflections of the high-pressure phase after correcting for the instrumental broadening gave $D_{V} \approx 16(6) \mathrm{nm}$, an order of magnitude smaller than the average size of crystallites of the low-pressure phase before the transition.

Upon release of pressure to below $1.1 \mathrm{GPa}$, the highpressure phase $\mathrm{V}$ of $\mathrm{Cd}_{3} \mathrm{As}_{2}$ converts back into the ambientcondition tetragonal structure. The phase transformation is complete at $P \approx 0.7 \mathrm{GPa}$. While the peak positions of the tetragonal phase I have been recovered, the peak widths and shapes recorded after the pressure cycling are remarkably different, as shown in Fig. 5. A strong perturbation of the relative peak intensities is evident especially in the range of $3^{\circ} \leqslant$ $2 \theta \leqslant 7^{\circ}$. Furthermore, the diffraction peaks of the ambientpressure phase recovered after releasing pressure are much broader compared with the pristine sample. For instance, the (224) reflection shown in the inset of Fig. 5(a) is broadened to nearly twice its original full width at half maximum. Size and strain analyses based on Le Bail refinements of powder XRD patterns recorded after reducing pressure to $0.2-0.7 \mathrm{GPa}$ indicated that the broadening of diffraction peaks from the low-pressure phase of $\mathrm{Cd}_{3} \mathrm{As}_{2}$ is dominated by microstrain. The best fits to the experimental data were achieved assuming Gaussian distribution describing the microstrain $\epsilon=$ $0.57(2) \%$ and a small Lorentzian or Gaussian contribution 


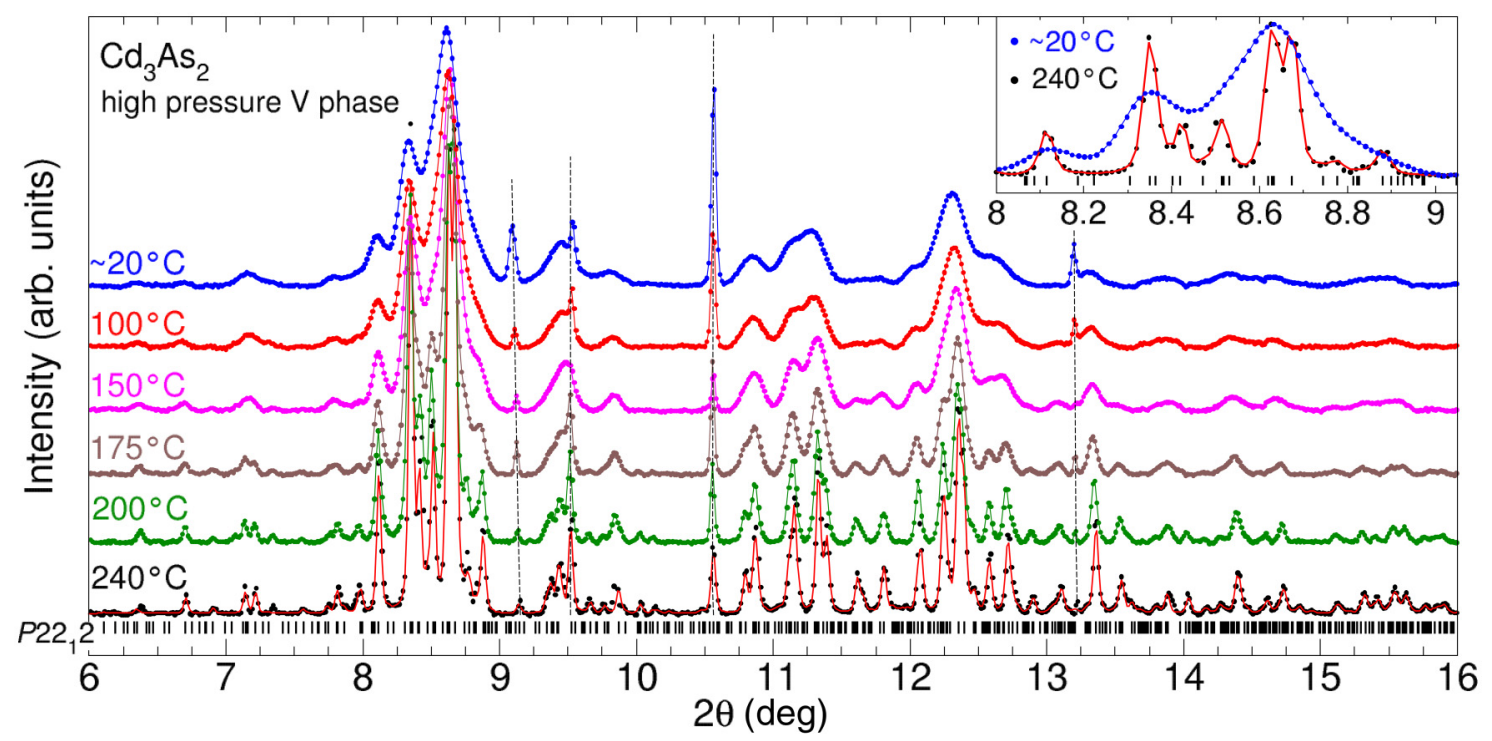

FIG. 6. XRD patterns for the high-pressure phase $\mathrm{V}$ of $\mathrm{Cd}_{3} \mathrm{As}_{2}$ measured on heating from room temperature to $240^{\circ} \mathrm{C}$ during which the load was kept constant on the DAC but pressure has increased from $4.18(5) \mathrm{GPa}$ at room temperature to $\sim 6 \mathrm{GPa}$ at $240^{\circ} \mathrm{C}$. Thick solid red lines represent Le Bail refinement performed assuming pseudo-Voigt profiles for diffraction peaks consistent with an orthorhombic lattice with the space group $P 22_{1} 2$ and the lattice parameters $a_{\mathrm{HP}}=8.68 \AA, b_{\mathrm{HP}}=17.15 \AA$, and $c_{\mathrm{HP}}=18.58 \AA$. Angular positions of diffraction peaks expected for this orthorhombic lattice are indicated by short vertical black lines. Dashed vertical lines show positions of diffraction peaks originating in $\mathrm{Cd}$. Inset shows enlarged angular range with the most intense peak manifold at room temperature (blue dots) and after annealing at $240^{\circ} \mathrm{C}$ (black dots) normalized to the maximum intensity. The XRD pattern of the annealed sample revealed five intense Bragg peaks for $2 \theta$ between $8.2^{\circ}$ and $8.7^{\circ}$, whereas for the $P 2_{1} / c$ structure proposed by Zhang et al. [29], only two Bragg reflections are expected in this angular range.

included to account for broadening due to the average coherent crystallite size $D_{V}=140(60) \mathrm{nm}$ similar to that before the pressure cycling. Noteworthy, a detailed inspection of the diffraction patterns measured after releasing pressure revealed that peak broadening is anisotropic and shows a distinct asymmetry, e.g., (116) and (316) peaks have pronounced tails on the low angle sides, indicated by arrows in Fig. 5(b). Both the asymmetry and $h k l$ dependence of peak profiles are typical for systems with a large degree of microstrain arising from high concentrations of dislocations and dislocation-type lattice defects [48-51]. In summary, the analysis of the peak widths provides evidence for large nonuniform strain and reduced crystallite domain size in the ambient-condition phase I of $\mathrm{Cd}_{3} \mathrm{As}_{2}$ recovered after the pressure cycling.

The high-pressure phase of $\mathrm{Cd}_{3} \mathrm{As}_{2}$ can be annealed at temperatures between $\sim 150$ and $240{ }^{\circ} \mathrm{C}$. The evolution of the XRD patterns collected a few minutes apart during heating is shown in Fig. 6. Upon increasing the temperature, the positions of diffraction peaks remain virtually unchanged due to a cancelation of thermal expansion and further compression as a result of the increasing pressure in the cell with friction locking the membrane. Most notably, raising the temperature above $\sim 150{ }^{\circ} \mathrm{C}$ leads to a systematic narrowing of the diffraction peaks within minutes between measurements. The process of decreasing the peak widths accelerates with increasing temperature, as expected for a recrystallization anneal and grain growth [52]. It ends shortly after the temperature of $200{ }^{\circ} \mathrm{C}$ is reached. The WilliamsonHall analyses (not shown) performed on several diffraction patterns recorded at temperatures between 230 and $240{ }^{\circ} \mathrm{C}$ on samples 2 and 3 indicated that, after the annealing, for both samples the microstrain was not larger than $\sim 0.06(\%)$, whereas the volume-weighted domain size increased from $\sim 16(6) \mathrm{nm}$ before the heat treatment to $\sim 80(15) \mathrm{nm}$ after the annealing.

The crystal structure of the high-pressure phase of $\mathrm{Cd}_{3} \mathrm{As}_{2}$ remains elusive, but XRD patterns recorded on the annealed samples reveal more details about the symmetry of the crystal lattice. The previously suggested monoclinic lattice $\left(P 2_{1} / c\right.$ [29]) is incompatible with the observed XRD patterns for the high-pressure phase $\mathrm{V}$ of $\mathrm{Cd}_{3} \mathrm{As}_{2}$ after the annealing. In particular, for the $P 2_{1} / c$ structure proposed by Zhang et al. [29], only two reflections are expected to contribute to the most intense peak manifold (see Fig. 1(d) in Ref. [29]), whereas the diffraction patterns of the annealed samples revealed five intense Bragg peaks in the angular range of $8.2^{\circ}$ to $8.7^{\circ}$, as shown in Fig. 6. Further, two Bragg peaks were detected at $2 \theta \approx 8.76^{\circ}$ and $8.88^{\circ}$, while no reflections are expected for the $P 2_{1} / c$ structure in this angular range. The XRD patterns measured on samples 2 and 3 after the annealing at temperatures up to $240^{\circ} \mathrm{C}$ at $P=5$ (1) GPa were virtually identical. Bragg reflections detected consistently in those diffraction patterns were used for the purpose of indexing. Searching for candidate crystal lattices did not give satisfactory results for cubic, tetragonal, hexagonal, and trigonal crystal systems with unit cell volumes of up to $6000 \AA^{3}$. Among orthorhombic structures, the best indexing solution with the smallest unit cell volume that accounts for the observed diffraction peaks has a primitive lattice with $a_{\mathrm{HP}} \approx 8.68 \AA, b_{\mathrm{HP}} \approx$ $17.15 \AA$, and $c_{\mathrm{HP}} \approx 18.58 \AA$. We note that such a crystal 


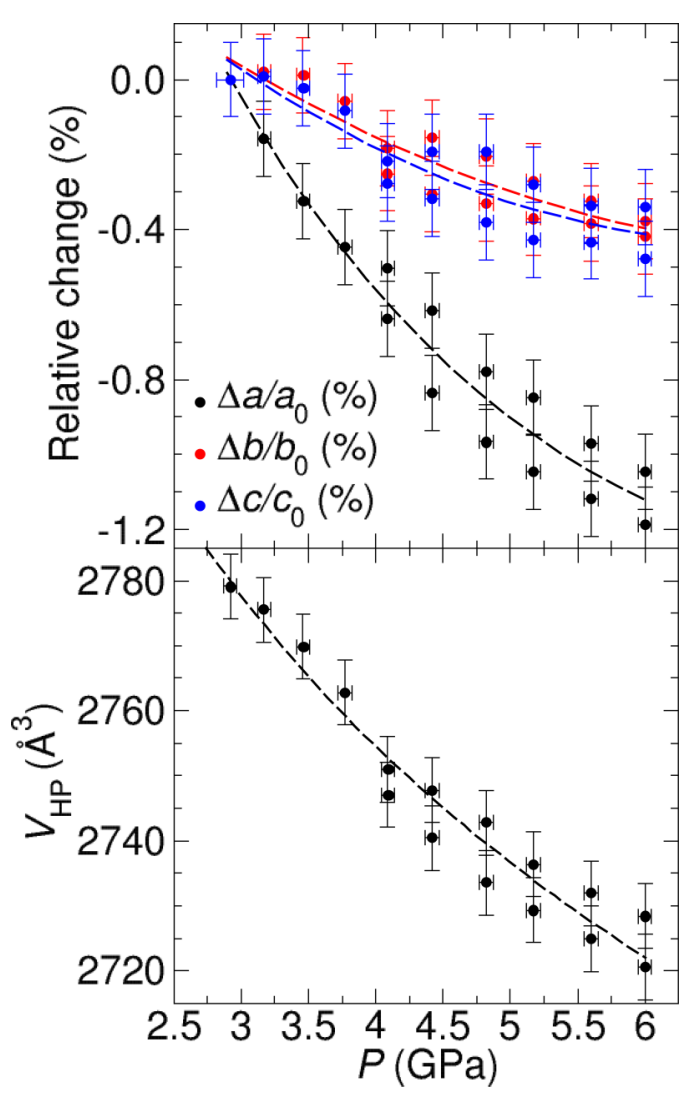

FIG. 7. The pressure dependence of the unit cell volume for the high-pressure phase $\mathrm{V}$ of $\mathrm{Cd}_{3} \mathrm{As}_{2}$ (bottom panel) and the pressureinduced relative changes in its lattice parameters (top panel) recorded at room temperature. Dashed lines are guides to the eye.

lattice may be considered as a superstructure of the antifluorite structure type made of 96 distorted $\mathrm{Cd}_{6} \square_{2}$ cubes, similarly to the ambient-pressure polymorphs. Searching for monoclinic cells gave candidate lattices with comparable or only slightly smaller unit cell volumes $(\Delta V / V \lesssim 12 \%)$. Notably, none of the lattices with smaller unit cell volumes could be interpreted in terms of the antifluorite-type building blocks. Therefore, we conclude that the structure of the high-pressure phase of $\mathrm{Cd}_{3} \mathrm{As}_{2}$ is likely primitive orthorhombic. Space group tests indicated the chiral $P 22_{1} 2$ or one of the lattices without systematic extinctions, i.e., centrosymmetric Pmmm, noncentrosymmetric chiral P222 or achiral $P 2 m m, P m 2 m$, or Pmm2. Indexing of the high-pressure $\mathrm{Cd}_{3} \mathrm{As}_{2}$ phase $\mathrm{V}$ assuming the space group $P 22_{1} 2$ is shown in Fig. 6.

To evaluate the compressibility of the high-pressure phase of $\mathrm{Cd}_{3} \mathrm{As}_{2}$, sequential Le Bail refinements of powder XRD patterns collected at room temperature were performed assuming the orthorhombic crystal lattice. The resulting pressure dependencies of the lattice parameters and the unit cell volume are shown in Fig. 7. The isothermal compressibility of the high-pressure phase immediately after the structural transformation is found to be $\kappa_{\mathrm{T}}=0.9(1) \times 10^{-11} \mathrm{~Pa}, 45 \%$ smaller than the compressibility of phase I $\left[\kappa_{T}=1.65(2) \times\right.$ $10^{-11} \mathrm{~Pa}$, see Fig. 4], and it decreases further with pressure. The notable reduction in compressibility can be rationalized by an increase in the density of $\mathrm{Cd}_{3} \mathrm{As}_{2}$ at the structural transition by $\sim 4.8(2) \%$ estimated from the lattice parameters of the low- and high-pressure phases, assuming that the latter is orthorhombic and consists of 96 distorted $\mathrm{Cd}_{6} \square_{2}$ cubes.

Upon lowering pressure below $\sim 1 \mathrm{GPa}$, the diffraction peaks of the annealed high-pressure phase in sample 3 start to diminish, and finally, the reflection pattern characteristic for the low-pressure phase of $\mathrm{Cd}_{3} \mathrm{As}_{2}$ emerges. As shown in Fig. 5, the broadening of the diffraction peaks is very similar to that observed for sample 1 after the pressure cycling at room temperature. However, next to the peaks expected for the ambient-condition phase of $\mathrm{Cd}_{3} \mathrm{As}_{2}$, there are also distinct additional contributions to the diffraction patterns at $2 \theta \approx 8.25^{\circ}, 8.78^{\circ}, 9.89^{\circ}$, and $11.80^{\circ}$ that may indicate the formation of a new phase. While intensities of those extra peaks vary between different sample spots, their angular positions remain the same. We note that the additional features correspond well to the diffraction peaks observed for sample 2 after annealing at temperatures up to $240{ }^{\circ} \mathrm{C}$ at pressures of $6 \mathrm{GPa}$ and releasing the pressure. Since for sample 2 glycerol was used as a pressure-transmitting medium causing significant deviations from hydrostatic conditions in the accessed $T-P$ regime $[53,54]$, we surmise that deviatoric stress induced by both the nonhydrostaticity of the pressure medium and larger size of the high-pressure phase crystallites can stabilize an intermediate metastable phase of $\mathrm{Cd}_{3} \mathrm{As}_{2}$ forming during the pressure-driven structural phase transition at room temperature.

The diffraction peaks of the intermediate phase of $\mathrm{Cd}_{3} \mathrm{As}_{2}$ observed at ambient conditions can be indexed as a primitive orthorhombic structure with the lattice parameters $a_{\mathrm{IP}}=$ $8.026(2) \AA, b_{\mathrm{IP}}=4.1145(5) \AA$, and $c_{\mathrm{IP}}=18.988(1) \AA$ and the space group $P 22_{1} 2_{1} 2$. We note that those lattice parameters are related to the lattice parameters of the highpressure phase: $a_{\mathrm{IP}} \approx a_{\mathrm{HP}}, b_{\mathrm{IP}} \approx b_{\mathrm{HP}}$, and $c_{\mathrm{IP}} \approx c_{\mathrm{HP}} / 4$, with the differences of $+7.7 \%,-2.2 \%$, and $+3.9 \%$, respectively. Therefore, we suspect that the intermediate phase of $\mathrm{Cd}_{3} \mathrm{As}_{2}$ can be considered as another superstructure of the antifluorite structure type. Le Bail refinements performed assuming pseudo-Voigt profiles for Bragg peaks compatible with the orthorhombic lattice indicated a sizable degree of nonuniform strain $\epsilon=0.16(2) \%$. The average crystallite size $D_{V}=108(10) \mathrm{nm}$ estimated from the refinements is comparable with the mean size of coherent domains of the high-pressure phase after annealing at temperatures of up to $240^{\circ} \mathrm{C}$.

\section{DISCUSSION}

Our high-pressure studies show that, for bulk $\mathrm{Cd}_{3} \mathrm{As}_{2}$, the phase I hosting topologically protected electronic states can be maintained up to $2.4 \mathrm{GPa}$ but cannot be recovered with similar high mobilities once this level of compression is exceeded. Recently, Goyal et al. [24] found that the quantum Hall effect associated with the surface states persists in thin films of $\mathrm{Cd}_{3} \mathrm{As}_{2}$ under strain up to $0.5 \%$. While Goyal et al. use biaxial strain in the (112) plane, the magnitude of the strain is equivalent to the modification of the crystal parameters at $1 \mathrm{GPa}$ in our work using hydrostatic pressure. Hence, we expect that thin films with strain above $\approx 1 \%$ will fall outside 
the stability range of $\mathrm{Cd}_{3} \mathrm{As}_{2}$ phase I and will no longer host quantum Hall effect states.

Our high-resolution powder XRD measurements allow us to evaluate in detail the changes in the lattice parameters of the ambient-condition tetragonal phase $\mathrm{I}$ of $\mathrm{Cd}_{3} \mathrm{As}_{2}$ induced by the applied pressure. While the observed pressure dependence of the unit cell volume shown in Fig. 4 is similar to those reported in Refs. [29,33], our XRD data does not give any indication for a sudden jump in $c / a$ at pressures below 1.5 GPa which Zhang et al. attributed to an additional topological phase transition [33]. Instead, our XRD study reveals a smooth decrease of the $c / a$ ratio with applied pressure. Remarkably, the observed changes in the $c / a$ ratio accord well with the pressure dependence of the Fermi wave vector derived from Shubnikov-de Haas oscillation measurements [33]. Both the axial compression along the $c$ direction and the unexpected shrinking of the Fermi surface accelerate at $P \gtrsim 1.7 \mathrm{GPa}$. First-principles electronic band structure calculations performed by Zhang et al. [33] indicated that the shortening of the lattice parameter $c$ should shift the Dirac nodes toward the center of the Brillouin zone, thus reducing the Fermi wave vector and providing an explanation for the gradual increase of resistivity under pressure within the ambient-condition phase of $\mathrm{Cd}_{3} \mathrm{As}_{2}$ (see Fig. 2(a) and Ref. [29]).

Our high-pressure studies provide a confirmation that the $\mathrm{I}-\mathrm{V}$ transition in $\mathrm{Cd}_{3} \mathrm{As}_{2}$ is of first-order type. The electrical resistivity and powder XRD measurements both detect the phase transition with an onset at $\sim 2.4$ and $\sim 1.3 \mathrm{GPa}$ on raising and releasing pressure, respectively. The sizable hysteresis evidences the first-order nature of the structural transformation, which shows a significant width of $\sim 0.2$ and $\sim 0.5 \mathrm{GPa}$ at $P_{c \uparrow}$ and $\sim 0.4$ and $\sim 0.5 \mathrm{GPa}$ at $P_{c \downarrow}$ based on the electrical resistivity and XRD data, respectively. We note that some discrepancies between the two methods are not unusual because the former potentially probes a percolative path, whereas the latter reflects changes in the entire sample volume. Importantly, the distinct pressure ranges with coexistence of the low- and high-pressure phases indicate sluggishness and thus an energy barrier between phases I and $\mathrm{V}$ of $\mathrm{Cd}_{3} \mathrm{As}_{2}$. Therefore, we deduce that the transition process involves drastic changes in the system of chemical bonds and leads to a major reorganization of the crystal structure.

The observed increase in width of diffraction peaks at the transition from phase I to phase $\mathrm{V}$ indicates that the transformation causes fragmentation of crystallites into much smaller domains. Therefore, we attribute the tenfold reduction in mobilities of charge carriers inferred from the electrical transport study to the combined effects of an increased scattering resulting from the strongly reduced coherent domain size and the loss of Dirac electrons with high Fermi velocity caused by changes in the symmetry of the crystal lattice overcompensating the increase in charge carrier concentration.

He et al. have interpreted the superconductivity observed above $8 \mathrm{GPa}$ to retain topological character [30]. This identification of topological superconductivity was based on presence of Dirac electrons with topological superconductivity possibly stabilized by a reduction of the crystal symmetry in the high-pressure phase [55]. However, our identification of the reconstructive I-V phase transition suggests a fundamental modification of the structure. While we show that the highpressure phase may contain the same $\mathrm{Cd}_{6} \square_{2}$ building blocks, they are very likely distorted and arranged in a different configuration. Hence, the Dirac electrons which are sensitive to the orbitals at the voids [22] are likely absent in the highpressure phase $\mathrm{V}$, and superconductivity in this phase is not of topological character. At the same time, our high-temperature annealing experiments highlight a route to study the superconductivity in this phase in more detail for samples with high crystallinity.

Recovering the ambient-condition phase I after cycling pressure at room temperature beyond $P_{c \uparrow}$ resulted in a large degree of microstrain. Estimates from profile refinements using the Le Bail method gave $\epsilon \approx 0.57(2) \%$, which is three orders of magnitude larger than $\epsilon$ values $\sim 10^{-6}$ expected for nearly strainfree crystals [56] and nearly three times larger than the microanstrain induced by grinding crystals into a fine powder with tens of nanometer sized grains. Although results of the size and strain analyses need to be considered with caution because of significant peak overlap, the large positive $\epsilon$ indicates the presence of local compressive stress fields arising from lattice disorder such as dislocations, stacking faults, intercalations of atoms, or grain boundaries. Such defects may give rise to unintentional doping [57,58], providing a plausible explanation for the observed decrease in the Hall coefficient for the ambient-condition phase I of $\mathrm{Cd}_{3} \mathrm{As}_{2}$ after the pressure cycling [see Fig. 2(b)]. Thus, we infer that the unexpected increase in resistivity after returning to the low-pressure regime below $P_{c \downarrow}$ shown in Fig. 2(a) originates in a largely enhanced scattering that outweighs the expected increase in carrier mobilities due to the formation of bulk Dirac points and the gain in the charge carriers from self-doping. We note that studies on $\mathrm{Cd}_{3} \mathrm{As}_{2}$ epilayers revealed a substantial effect of extended defects on electron mobilities [59]. Moreover, for epitaxial (112) $\mathrm{Cd}_{3} \mathrm{As}_{2}$ films, a systematic decrease in Hall mobilities with the compressive in-plane strain $\left(\epsilon_{\mathrm{s}} \lesssim 0.5 \%\right)$ was observed [24]. Further studies, including high-resolution transmission electron microscopy imaging, are needed to address the origin of the increased scattering in the ambient-condition phase of $\mathrm{Cd}_{3} \mathrm{As}_{2}$ retrieved after the room-temperature pressure cycling.

\section{CONCLUSIONS}

Our high-pressure studies highlight the disruptive nature of the pressure-driven structural phase transition in $\mathrm{Cd}_{3} \mathrm{As}_{2}$. We find large changes in both the electronic and microstructural characteristics at the room-temperature structural transformation. The reduction of the carrier mobility is shown to result from the formation of microstrain and fragmentation of crystallites into smaller domains caused by the phase transition. Our transport measurements indicate that the increased resistance is dominated by the enhanced scattering outweighing the gain in charge carriers in the high-pressure phase V. We show that annealing of the highpressure phase at temperatures of $\sim 200{ }^{\circ} \mathrm{C}$ results in a largely improved crystallinity and thus should enable future studies aiming at solving the crystal structure and exploring 
the electronic properties. Finally, we observe the formation of a new primitive orthorhombic phase prompted presumably by deviatoric stress upon releasing pressure through the structural phase transition. Our results highlight the importance of considering strain and crystallite size when tailoring electronic properties of $\mathrm{Cd}_{3} \mathrm{As}_{2}$ for applications of bulk and thin films.

Data are available at the University of Bristol data repository data.bris [60].

\section{ACKNOWLEDGMENTS}

The authors would like to thank Ingo Loa for valuable discussion and Charles Clapham and Chris Bell for technical support. This paper was partially supported by the EPSRC under Grants No. EP/R011141/1, No. EP/L025736/1, No. EP/N026691/1, as well as the ERC Horizon 2020 programme under Grant No. 715262-HPSuper. We acknowledge Diamond Light Source for time on beamline I15 under proposal EE19319-1.
[1] N. P. Armitage, E. J. Mele, and A. Vishwanath, Weyl and Dirac semimetals in three-dimensional solids, Rev. Mod. Phys. 90, 015001 (2018).

[2] Z. Wang, H. Weng, Q. Wu, X. Dai, and Z. Fang, Threedimensional Dirac semimetal and quantum transport in $\mathrm{Cd}_{3} \mathrm{As}_{2}$, Phys. Rev. B 88, 125427 (2013).

[3] Z. K. Liu, J. Jiang, B. Zhou, Z. J. Wang, Y. Zhang, H. M. Weng, D. Prabhakaran, S.-K. Mo, H. Peng, P. Dudin, T. Kim, M. Hoesch, Z. Fang, X. Dai, Z. X. Shen, D. L. Feng, Z. Hussain, and Y. L. Chen, A stable three-dimensional topological Dirac semimetal $\mathrm{Cd}_{3} \mathrm{As}_{2}$, Nat. Mater. 13, 677 (2014).

[4] S. Borisenko, Q. Gibson, D. Evtushinsky, V. Zabolotnyy, B. Büchner, and R. J. Cava, Experimental Realization of a ThreeDimensional Dirac Semimetal, Phys. Rev. Lett. 113, 027603 (2014).

[5] M. Neupane, S.-Y. Xu, R. Sankar, N. Alidoust, G. Bian, C. Liu, I. Belopolski, T.-R. Chang, H.-T. Jeng, H. Lin, A. Bansil, F. Chou, and M. Z. Hasan, Observation of a three-dimensional topological Dirac semimetal phase in high-mobility $\mathrm{Cd}_{3} \mathrm{As}_{2}$, Nat. Commun. 5, 3786 (2014).

[6] L. P. He, X. C. Hong, J. K. Dong, J. Pan, Z. Zhang, J. Zhang, and S. Y. Li, Quantum Transport Evidence for the ThreeDimensional Dirac Semimetal Phase in $\mathrm{Cd}_{3} \mathrm{As}_{2}$, Phys. Rev. Lett. 113, 246402 (2014).

[7] P. J. W. Moll, N. L. Nair, T. Helm, A. C. Potter, I. Kimchi, A. Vishwanath, and J. G. Analytis, Transport evidence for Fermiarc-mediated chirality transfer in the Dirac semimetal $\mathrm{Cd}_{3} \mathrm{As}_{2}$, Nature 535, 266 (2016).

[8] C. Zhang, A. Narayan, S. Lu, J. Zhang, H. Zhang, Z. Ni, X. Yuan, Y. Liu, J.-H. Park, E. Zhang, W. Wang, S. Liu, L. Cheng, L. Pi, Z. Sheng, S. Sanvito, and F. Xiu, Evolution of Weyl orbit and quantum Hall effect in Dirac semimetal $\mathrm{Cd}_{3} \mathrm{As}_{2}$, Nat. Commun. 8, 1272 (2017).

[9] C. Zhang, Y. Zhang, X. Yuan, S. Lu, J. Zhang, A. Narayan, Y. Liu, H. Zhang, Z. Ni, R. Liu, E. S. Choi, A. Suslov, S. Sanvito, L. Pi, H.-Z. Lu, A. C. Potter, and F. Xiu, Quantum Hall effect based on Weyl orbits in $\mathrm{Cd}_{3} \mathrm{As}_{2}$, Nature 565, 331 (2019).

[10] M. Uchida, Y. Nakazawa, S. Nishihaya, K. Akiba, M. Kriener, Y. Kozuka, A. Miyake, Y. Taguchi, M. Tokunaga, N. Nagaosa, Y. Tokura, and M. Kawasaki, Quantum Hall states observed in thin films of Dirac semimetal $\mathrm{Cd}_{3} \mathrm{As}_{2}$, Nat. Commun. 8, 2274 (2017).

[11] T. Schumann, L. Galletti, D. A. Kealhofer, H. Kim, M. Goyal, and S. Stemmer, Observation of the Quantum Hall Effect in
Confined Films of the Three-Dimensional Dirac Semimetal $\mathrm{Cd}_{3} \mathrm{As}_{2}$, Phys. Rev. Lett. 120, 016801 (2018).

[12] Y. Nakazawa, M. Uchida, S. Nishihaya, M. Kriener, Y. Kozuka, Y. Taguchi, and M. Kawasaki, Structural characterisation of high-mobility $\mathrm{Cd}_{3} \mathrm{As}_{2}$ films crystallised on $\mathrm{SrTiO}_{3}$, Sci. Rep. 8, 2244 (2018).

[13] C. Zhu, F. Wang, Y. Meng, X. Yuan, F. Xiu, H. Luo, Y. Wang, J. Li, X. Lv, L. He, Y. Xu, J. Liu, C. Zhang, Y. Shi, R. Zhang, and $\mathrm{S}$. Zhu, A robust and tuneable mid-infrared optical switch enabled by bulk Dirac fermions, Nat. Commun. 8, 14111 (2017).

[14] O. F. Shoron, M. Goyal, B. Guo, D. A. Kealhofer, T. Schumann, and S. Stemmer, Prospects of terahertz transistors with the topological semimetal cadmium arsenide, Adv. Electron. Mater. 6, 2000676 (2020)

[15] Q. Wang, C.-Z. Li, S. Ge, J.-G. Li, W. Lu, J. Lai, X. Liu, J. Ma, D.-P. Yu, Z.-M. Liao, and D. Sun, Ultrafast broadband photodetectors based on three-dimensional Dirac semimetal $\mathrm{Cd}_{3} \mathrm{As}_{2}$, Nano Lett. 17, 834 (2017).

[16] T. Liang, Q. Gibson, M. N. Ali, M. Liu, R. J. Cava, and N. P. Ong, Ultrahigh mobility and giant magnetoresistance in the Dirac semimetal $\mathrm{Cd}_{3} \mathrm{As}_{2}$, Nat. Mater. 14, 280 (2014).

[17] A. Narayanan, M. D. Watson, S. F. Blake, N. Bruyant, L. Drigo, Y. L. Chen, D. Prabhakaran, B. Yan, C. Felser, T. Kong, P. C. Canfield, and A. I. Coldea, Linear Magnetoresistance Caused by Mobility Fluctuations in $n$-Doped $\mathrm{Cd}_{3} \mathrm{As}_{2}$, Phys. Rev. Lett. 114, 117201 (2015).

[18] A. B. Davydov, L. N. Oveshnikov, A. V. Suslov, A. I. Ril, S. F. Marenkin, and B. A. Aronzon, Superconductivity in thin films of the Dirac semimetal $\mathrm{Cd}_{3} \mathrm{As}_{2}$, Phys. Solid State 62, 419 (2020).

[19] C. Zhang, E. Zhang, W. Wang, Y. Liu, Z.-G. Chen, S. Lu, S. Liang, J. Cao, X. Yuan, L. Tang, Q. Li, C. Zhou, T. Gu, Y. Wu, J. Zou, and F. Xiu, Room-temperature chiral charge pumping in Dirac semimetals, Nat. Commun. 8, 13741 (2017).

[20] C. Fu, Y. Sun, and C. Felser, Topological thermoelectrics, APL Mater. 8, 040913 (2020).

[21] M. N. Ali, Q. Gibson, S. Jeon, B. B. Zhou, A. Yazdani, and R. J. Cava, The crystal and electronic structures of $\mathrm{Cd}_{3} \mathrm{As}_{2}$, the threedimensional electronic analogue of graphene, Inorg. Chem. 53, 4062 (2014).

[22] A. Mosca Conte, O. Pulci, and F. Bechstedt, Electronic and optical properties of topological semimetal $\mathrm{Cd}_{3} \mathrm{As}_{2}$, Sci. Rep. 7, 45500 (2017).

[23] B. Yang and N. Nagaosa, Classification of stable threedimensional Dirac semimetals with nontrivial topology, Nat. Commun. 5, 4898 (2014). 
[24] M. Goyal, H. Kim, T. Schumann, L. Galletti, A. A. Burkov, and S. Stemmer, Surface states of strained thin films of the Dirac semimetal $\mathrm{Cd}_{3} \mathrm{As}_{2}$, Phys. Rev. Materials 3, 064204 (2019).

[25] C. W. F. T. Pistorius, Melting and polymorphism of $\mathrm{Cd}_{3} \mathrm{As}_{2}$ and $\mathrm{Zn}_{3} \mathrm{As}_{2}$ at high pressures, High Temp. High Press. 7, 441 (1975).

[26] A. Pietraszko and K. Lukaszewicz, Thermal expansion and phase transitions of $\mathrm{Cd}_{3} \mathrm{As}_{2}$ and $\mathrm{Zn}_{3} \mathrm{As}_{2}$, Phys. Status Solidi A 18, 723 (1973).

[27] E. K. Arushanov, Crystal growth and characterization of II3V2 compounds, Prog. Cryst. Growth Charact. 3, 211 (1980).

[28] M. D. Banus and M. C. Lavine, X-ray diffraction studies on $\mathrm{Zn}_{3} \mathrm{As}_{2}$ and $\mathrm{Cd}_{3} \mathrm{As}_{2}$ at high pressure, High Temp. High Press. 1, 269 (1969).

[29] S. Zhang, Q. Wu, L. Schoop, M. N. Ali, Y. Shi, N. Ni, Q. Gibson, S. Jiang, V. Sidorov, W. Yi, J. Guo, Y. Zhou, D. Wu, P. Gao, D. Gu, C. Zhang, S. Jiang, K. Yang, A. Li, Y. Li, X. Li, J. Liu, X. Dai, Z. Fang, R. J. Cava, L. Sun, and Z. Zhao, Breakdown of three-dimensional Dirac semimetal state in pressurized $\mathrm{Cd}_{3} \mathrm{As}_{2}$, Phys. Rev. B 91, 165133 (2015).

[30] L. P. He, Y. T. Jia, S. J. Zhang, X. C. Hong, C. Q. Jin, and S. Y. Li, Pressure-induced superconductivity in the threedimensional Dirac semimetal $\mathrm{Cd}_{3} \mathrm{As}_{2}$, Nat. Quantum Mater. 1, 16014 (2016).

[31] S. N. Gupta, D. V. S. Muthu, C. Shekhar, R. Sankar, C. Felser, and A. K. Sood, Pressure-induced electronic and structural phase transitions in Dirac semimetal $\mathrm{Cd}_{3} \mathrm{As}_{2}$ : Raman study, Europhys. Lett. 120, 57003 (2017).

[32] E. Uykur, R. Sankar, D. Schmitz, and C. A. Kuntscher, Optical spectroscopy study on pressure-induced phase transitions in the three-dimensional Dirac semimetal $\mathrm{Cd}_{3} \mathrm{As}_{2}$, Phys. Rev. B 97, 195134 (2018).

[33] C. Zhang, J. Sun, F. Liu, A. Narayan, N. Li, X. Yuan, Y. Liu, J. Dai, Y. Long, Y. Uwatoko, J. Shen, S. Sanvito, W. Yang, J. Cheng, and F. Xiu, Evidence for pressure-induced node-pair annihilation in $\mathrm{Cd}_{3} \mathrm{As}_{2}$, Phys. Rev. B 96, 155205 (2017).

[34] M. I. Eremets, High Pressure Experimental Methods (Oxford University Press, Oxford, New York, 1996).

[35] K. Syassen, Ruby under pressure, High Press. Res. 28, 75 (2008).

[36] J. Filik, A. W. Ashton, P. C. Y. Chang, P. A. Chater, S. J. Day, M. Drakopoulos, M. W. Gerring, M. L. Hart, O. V. Magdysyuk, S. Michalik, A. Smith, C. C. Tang, N. J. Terrill, M. T. Wharmby, and $\mathrm{H}$. Wilhelm, Processing two-dimensional $\mathrm{x}$-ray diffraction and small-angle scattering data in DAWN2, J. Appl. Crystallogr. 50, 959 (2017).

[37] S. N. Vaboya and G. C. Kennedy, Compressibility of 18 metals to 45 kbar, J. Phys. Chem. Solids 31, 2329 (1970).

[38] V. Petř́íček, M. Dušek, and L. Palatinus, Crystallographic computing system JANA2006: general features, Z. Kristallogr. 229, 345 (2014).

[39] The pseudo-Voigt function is an approximation of the Voigt function defined as

$$
p V(x)=\eta L(x)+(1-\eta) G(x),
$$

with $L(x)$ and $G(x)$ denoting Lorentzian and Gaussian parts, respectively, that are of the same full width at half maximum (FWHM) $H$, and are weighted by $\eta$ adopting values between 0 and 1 and thus shifting the profile more toward pure Gaussian or pure Lorentzian. The $H$ and $\eta$ parameters are related to the FWHM values of the deconvoluted Lorentzian $\left(H_{L}\right)$ and Gaussian $\left(H_{G}\right)$ functions [61]:

$$
\begin{aligned}
H= & H_{G}^{5}+2.69269 H_{G}^{4} H_{L}+2.42843 H_{G}^{3} H_{L}^{2}+4.47163 H_{G}^{2} H_{L}^{3} \\
& +0.07842 H_{G} H_{L}^{4}+H_{L}^{5}, \\
\eta= & 1.36603 \frac{H_{L}}{H}-0.47719\left(\frac{H_{L}}{H}\right)^{2}+0.11116\left(\frac{H_{L}}{H}\right)^{3} .
\end{aligned}
$$

[40] P. Scherrer, Bestimmung der Grösse und der inneren Struktur von Kolloidteilchen mittels Röntgenstrahlen, Nachr. Ges. Wiss. Göttingen 26, 98 (1918).

[41] J. I. Langford and A. J. C. Wilson, Scherrer after sixty years: a survey and some new results in the determination of crystallite size, J. Appl. Cryst. 11, 102 (1978).

[42] The integral breadths of the Lorentzian, Gaussian, and normalized pseudo-Voigt functions are related to their full width at half maximum (FWHM) as follows:

$$
\begin{gathered}
\beta_{L}=\frac{\pi H_{L}}{2}, \\
\beta_{G}=\frac{H_{G}}{2} \sqrt{\frac{\pi}{\ln 2}}, \\
\beta_{p V}=\frac{0.5 \pi H}{\eta+(1-\eta) \sqrt{\pi \ln 2}},
\end{gathered}
$$

respectively.

[43] G. K. Williamson and W. H. Hall, X-ray line broadening from filed aluminium and wolfram, Acta Metall. 1, 22 (1953).

[44] A. Le Bail, Monte Carlo indexing with McMaille, Powder Diffr. 19, 249 (2004).

[45] A. Boultif and D. Louër, Powder pattern indexing with the successive dichotomy method., J. Appl. Cryst. 37, 724 (2004).

[46] A. J. Rosenberg and T. C. Harman, $\mathrm{Cd}_{3} \mathrm{As}_{2}-$ a noncubic semiconductor with unusually high electron mobility, J. Appl. Phys. 30, 1621 (1959).

[47] G. A. Steigmann and J. Goodyear, The crystal structure of $\mathrm{Cd}_{3} \mathrm{As}_{2}$, Acta Cryst. B24, 1062 (1968).

[48] I. Groma, T. Ungár, and M. Wilkens, Asymmetric x-ray line broadening of plastically deformed crystals. I. Theory, J. Appl. Cryst. 21, 47 (1988).

[49] M. Krivoglaz, X-ray and Neutron Diffraction in Nonideal Crystals (Springer-Verlag, Berlin, 1996), pp. 357-420.

[50] T. Ungár, I. Groma, and M. Wilkens, Asymmetric x-ray line broadening of plastically deformed crystals. II. Evaluation procedure and application to [001]-Cu crystals, J. Appl. Cryst. 22, 26 (1989).

[51] I. Groma and G. Monnet, Analysis of asymmetric broadening of x-ray diffraction peak profiles caused by randomly distributed polarized dislocation dipoles and dislocation walls, J. Appl. Cryst. 35, 589 (2002).

[52] J. Humphreys, G. S. Rohrer, and A. Rollet, Recrystallization and Related Annealing Phenomena, Third Edition (Elsevier, Amsterdam, Netherlands, 2017).

[53] N. Tateiwa and Y. Haga, Evaluations of pressure-transmitting media for cryogenic experiments with diamond anvil cell, Rev. Sci. Instrum. 80, 123901 (2009).

[54] O. Moulding, I. Osmond, F. Flicker, T. Muramatsu, and S. Friedemann, Absence of superconducting dome at the chargedensity-wave quantum phase transition in $2 \mathrm{H}-\mathrm{NbSe}_{2}$, Phys. Rev. Research 2, 043392 (2020). 
[55] S. Kobayashi and M. Sato, Topological Superconductivity in Dirac Semimetals, Phys. Rev. Lett. 115, 187001 (2015).

[56] S. K. Kushwaha, K. K. Maurya, N. Vijayan, B. Kumar, R. Bhatt, S. Ganesamoorthy, and G. Bhagavannarayana, Crystalline perfection, Raman, UV-VIS-NIR and prism coupler investigations on $\mathrm{Cz}$-grown pure and $\mathrm{Zn}$-doped $\mathrm{LiNbC}_{3}$ single crystals, CrystEngComm 14, 3297 (2012).

[57] E. Dumiszewska, W. Strupinski, and K. Zdunek, Interaction between dislocations density and carrier concentration of gallium nitride layers, J. Superhard Mater. 29, 174 (2007).

[58] F. Hyuga, Effect of dislocations on sheet carrier concentration of Si-implanted, semi-insulating, liquid-encapsulated
Czochralski grown GaAs, Jpn. J. Appl. Phys. 24, L160 (1985).

[59] A. D. Rice, K. Park, E. T. Hughes, K. Mukherjee, and K. Alberi, Defects in $\mathrm{Cd}_{3} \mathrm{As}_{2}$ epilayers via molecular beam epitaxy and strategies for reducing them, Phys. Rev. Materials 3, 121201(R) (2019).

[60] S. Friedemann, Data for publication "Pressure-induced reconstructive phase transition in $\mathrm{Cd}_{3} \mathrm{As}_{2}$ ", http://dx.doi.org/10.5523/ bris.1boq1ta44ocxf2sdv5w11hagg2 (2021).

[61] P. Thompson, D. E. Cox, and J. B. Hastings, Rietveld refinement of Debye-Scherrer synchrotron x-ray data from $\mathrm{Al}_{2} \mathrm{O}_{3}$, J. Appl. Cryst. 20, 79 (1987). 